\title{
Identifying Non-Darcian Flow and Non-Fickian Pressure Propagation in Field-Scale Discrete Fracture Networks
}

\author{
Bingqing Lu1 ${ }^{1}$, Yong Zhang1 ${ }^{*}$, Yuan Xia ${ }^{2}$, Donald M. Reeves ${ }^{3}$, \\ Hongguang Sun ${ }^{4}$, Dongbao Zhou ${ }^{4}$, Chunmiao Zheng 5 \\ ${ }^{1}$ Department of Geological Sciences, University of Alabama, Tuscaloosa, Alabama, USA \\ ${ }^{2}$ Colllege of Environmental Science and Engineering, Guilin University of Technology, Guilin, China \\ ${ }^{3}$ Department of Geological and Environmental Sciences, Western Michigan University, Kalamazoo, Michigan, USA \\ ${ }^{4}$ College of Mechanics and Materials, Hohai University, Nanjing, China \\ ${ }^{5}$ School of Environmental Science \& Engineering, Southern University of Sciences and Technology, Shenzhen, China \\ Email: *yzhang264@ua.edu
}

How to cite this paper: Lu, B.Q., Zhang, Y., Xia, Y., Reeves, D.M., Sun, H.G., Zhou, D.B. and Zheng, C.M. (2018) Identifying Non-Darcian Flow and Non-Fickian Pressure Propagation in Field-Scale Discrete Fracture Networks. Journal of Geoscience and Environment Protection, 6, 59-69. https://doi.org/10.4236/gep.2018.65005

Received: March 13, 2018

Accepted: May 20, 2018

Published: May 23, 2018

\begin{abstract}
Non-Darcian flow has been well documented for fractured media, while the potential non-Darcian flow and its driven factors in field-scale discrete fracture networks (DFNs) remain obscure. This study conducts Monte Carlo simulations of water flow through DFNs to identify non-Darcian flow and non-Fickian pressure propagation in field-scale DFNs, by adjusting fracture density, matrix hydraulic conductivity, and the general hydraulic gradient. Numerical simulations and analyses show that interactions of the fracture architecture with the hydraulic gradient affect non-Darcian flow in DFNs, by generating and adjusting complex pathways for water. The fracture density affects significantly the propagation of hydraulic head/pressure in the DFN, likely due to fracture connectivity and flow channeling. The non-Darcian flow pattern may not be directly correlated to the non-Fickian pressure propagation process in the regional-scale DFNs, because they refer to different states of water flow and their controlling factors may not be the same. Findings of this study improve our understanding of the nature of flow in DFNs.
\end{abstract}

\section{Keywords}

Discrete Fracture Networks, Non-Darcian Flow, Non-Fickian Pressure Propagation

\section{Introduction}

Darcy's law proposed by Henry Darcy maintains that the specific discharge of 
water increases linearly with the gradient of hydraulic head along a 3.5-m-long saturated column filled with homogeneous sand [1]. This fundamental law has been used to quantify various dynamics in natural media with different degrees of heterogeneity and scales for more than one century, such as disposal of radioactive waste, geothermal utilization by hot dry rock systems, oil and gas production from fractured reservoirs, and water production from fractured rock [2] [3]. However, subsurface fluid flow with non-Darcian characteristics, where the flow rate is nonlinearly related to the hydraulic gradient (also called "pressure drop"), has been detected in fractured media for decades, especially in the petroleum industry [4] which noted that quantifying these effects has proved difficult [5]. For example, non-Darcian flow has been observed in a variety of situations, such as a single confined vertical fracture toward a well [6], catalytic packed-bed reactors [7], and fractured rock [2].

This study aims at exploring the potential for non-Darcy flow in field-scale discrete fracture networks (DFNs). Fluid flow transition from Darcian to non-Darcian has been confirmed and broadly studied in the case of a single rock fracture [8], while such a transition in DFNs has not been fully studied. In addition, non-Darcy flow can occur over a broad spectrum of flow rates, including turbulent flow (for Reynolds number larger than 2000) and low rates in the initial regime (due to the competition between the interface friction and the pressure gradient). For example, previous studies found a nonlinear relationship between flow rate and pressure drop when either of these parameters becomes large [9] [10]. This study investigates the impact of flow velocity characteristics of subsurface flow through fracture networks on the evolution of non-Darcy dynamics. To address the above issues, we will conduct flow simulations involving DFNs with systematic changes in geometric characteristics and applied hydraulic gradient. The impacts of fracture density and matrix permeability are also studied.

We will also explore the possible impact of non-Darcy flow on the transient dynamics of water flow through DFNs, which has not been addressed in previous studies. Darcy or non-Darcy flow is usually defined using steady-state flows, where the asymptotic flow rate is used to build the relationship with the pressure drop. Water flow in natural aquifers is often transient, due to the change of input (such as short-term weather change) and/or output (i.e., pumping), and therefore the transient flow dynamics are practically important.

The rest of this work is organized as follows. Section 2 presents the Monte Carlo approach to simulation water flux and pressure propagation through multiple DFNs, which is one of the most efficient ways to investigate the impact of fracture properties on water flow behaviors. Results of the Monte Carlo simulations and evaluation using a standard dispersion equation (SDE) are presented in section 3. In section 4, we discuss the possible signal of non-Darcian flow and non-Fickian pressure propagation and their characterizations. The impacts of fracture density and rock matrix permeability on non-Darcian flow and non-Fickian pressure transfer are investigated. Conclusions are drawn in 
section 5 .

\section{Monte Carlo Simulation and Standard-Dispersion Equation}

There are three major steps in the Monte Carlo simulation of water flow through saturated field-scale DFNs. First, we generate equally possible but different realizations of stochastic fracture networks for DFNs for each pre-assigned fracture density, using Hydro Geo Sphere (HGS) software (v.111, Aquanty Inc., Waterloo, ON, Canada) [11]. HGS is a multi-dimensional, control-volume, finite element simulator designed to quantify the hydrologic cycle, including groundwater flow and transport in fractured aquifers. Second, pressure drop and water flow through the generated DFNs under both steady state and transient conditions are modeled using HGS, providing the synthetic data to evaluate the influence of the DFN property on water flow dynamics. Third, propagation of the hydraulic head/pressure is calculated by the Fick's law-based dispersion equation, which describes the Fickian type of transport and can be used to identify any anomalous dynamics embedded in transient flow in complex DFNs.

\subsection{Random Discrete Fracture Network Generation}

The two-dimensional DFN has a dimension of $50 \mathrm{~m}$ (discretized into 100 blocks) along the longitudinal direction ( $x$ axis) and $25 \mathrm{~m}$ (50 blocks) vertically ( $z$ axis). Three scenarios of DFNs, with each containing 100 realizations of DFNs, are built, which have their own unique time-dependent seed based on the current system time to generate random fractures. Each fracture network is composed of two superimposed sets of fractures, which are orthogonal to each other (orientations are $0^{\circ}$ and $90^{\circ}$ ) as observed commonly in realistic DFNs [12]. The ensemble average of the 100 flow and pressure propagation simulations are calculated for each scenario. A similar geometry was used previously to study subdiffusive transport in fractured formations by Lu et al. [13], where details about the DFN models, such as fracture locations, orientation, length, and hydraulic conductivities distributions can be found. We extend the work of Lu et al. [13] by investigating the relationship between pressure drop and flow rate, one of the fundamental problems in hydrologic sciences. We also test the influence of matrix hydraulic conductivity $K$, which increases from $1 \times 10^{-8}$ to $1 \times 10^{-7} \mathrm{~m} / \mathrm{s}$.

\subsection{Modeling Groundwater Flow and Pressure Propagationin DFNs}

Both the steady-state and transient groundwater flow through the confined aquifer generated above were solved by HGS. Parameters for the flow model are the same as those used in [13], which are chosen based on field experiments and literature values [14] [15]. The main flow direction is from left to right, with a general hydraulic gradient $(\lambda)$ defined as the ratio of the hydraulic head difference to the DFN domainsize $(\Delta x=50 \mathrm{~m})$. The left and right boundaries are then assigned as constant head boundaries, to propagate the general hydraulic gra- 
dient across the model domain.

\subsection{Standard-Dispersion Equation to Quantify Pressure Propagation}

Hydraulic head (or the propagation of the hydraulic pressure) is typically described by the well-known Boussinesq flow equation [16], which can be written in the following standard-diffusion equation form with constant parameter:

$$
\frac{\partial p}{\partial t}=D \frac{\partial^{2} p}{\partial x^{2}}
$$

where $p(x, t)$ denotes the spatially and temporally varying pressure, and $D$ represents the diffusion coefficient. Considering the initial condition: $p(x, t=0)$ $=0$ and the constant-pressure boundary conditions $\left(p(x=0, t=0)=p_{l}\right.$ for the inlet boundary), we obtain the following analytical solution for model (1):

$$
p(x, t)=p_{l}\left[1-\operatorname{erf}\left(\frac{x}{\sqrt{4 D t}}\right)\right],
$$

where $\operatorname{erf}(\cdot)$ represents the error function.

In the following two sections, we will model the pressure propagation using the $\operatorname{SDE}(1)$ to investigate the mechanism behind the propagation of the pressure (head).

\section{Results of Monte Carlo Simulations}

The ensemble average of steady-state water flux across the outlet (right) boundary for all 100 realizations is plotted in Figure 1 with the pre-assigned hydraulic gradient ( $/$ ). Curves in Figure 1 show the best-fit linear trendline (black line) and the power-law trendline (blue line). Three scenarios of DFNs with different fracture densities are considered, which contain 20,60 and 100 fractures, respectively.

Results show that the DFN with 100 fractures contains the largest noise in the simulated flux (especially for a relatively small hydraulic gradient: $1 \times 10^{-6}<J<$ $1 \times 10^{-3}$ ), and the relationship between hydraulic gradient and flux transfers from non-linear (power-law) to linear gradually (Figure 1(c)). The noise in Figure 1(c) might be an artificial oscillation due to the solver or the limited number of realizations, but the nonlinear region may be real since it also appears in all the other scenarios (Figure 1(a), Figure 1(b)). With less fractures in the rock mass, the nonlinear portion shortens (Figure 1(a), Figure 1(b)). In all scenarios, the overall increasing trend of water flux due to an increasing hydraulic gradient can be captured by a power-law function (Figure 1), satisfying the Izbash law [17].

The simulated transient flux at the outlet boundary is shown in Figure 2 with the matrix hydraulic conductivity $K=1 \times 10^{-7} \mathrm{~m} / \mathrm{s}$. For illustration purposes, here we show the results with three hydraulic gradients: $J=1 \times 10^{-5}, 1 \times 10^{-4}$, and $1 \times 10^{-3}$. To explore the impact of $K$ on transient flux, we re-ran the above Monte Carlo simulations and obtain the transient flux for $K=1 \times 10^{-8} \mathrm{~m} / \mathrm{s}$ (Figure 3). The best-fit of transient flux using the SDE (1) is also shown in these figures. 
(a) Flux with 20 fractures in a log-log plot

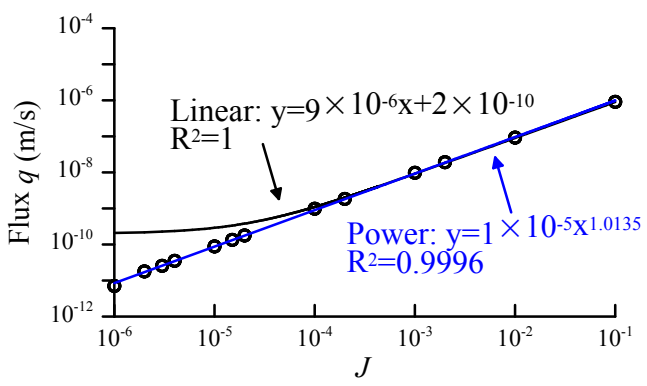

(c) Flux with 100 fractures in a log-log plot

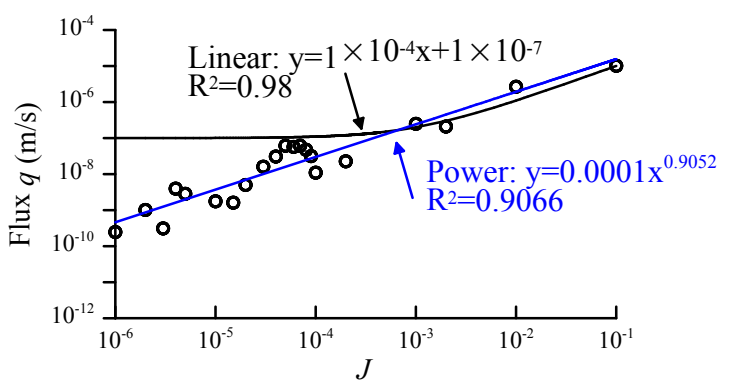

(b) Flux with 60 fractures in a log-log plot

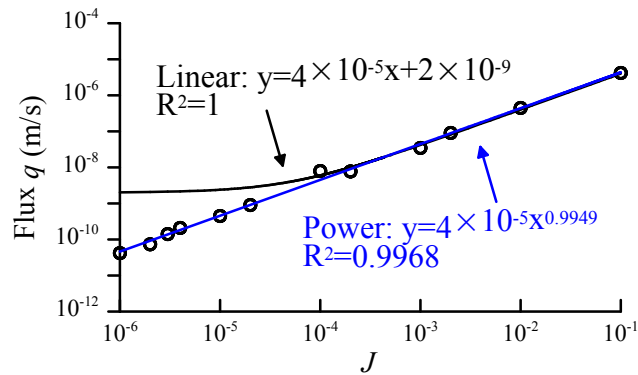

Figure 1. Ensemble average flux at the outlet boundary for the three DFN scenarios with a different number of fractures: 20 (a), 60 (b), and 100 (c). The symbols are steady-state flux data simulated by HGS, and the solid lines are the best-fit trendlines.

(a) Flux with 20 fractures in a semi-log plot

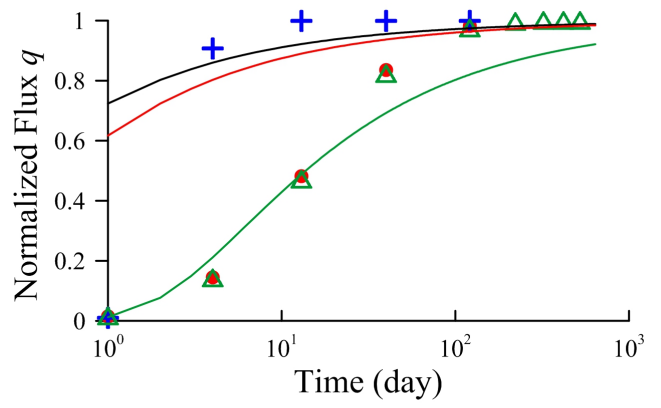

(c) 100 fractures

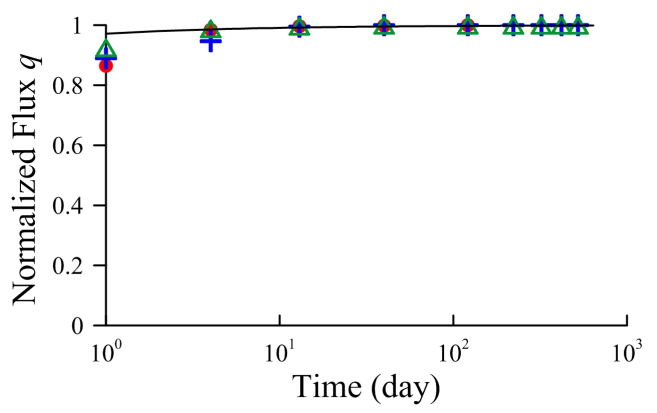

(b) 60 fractures

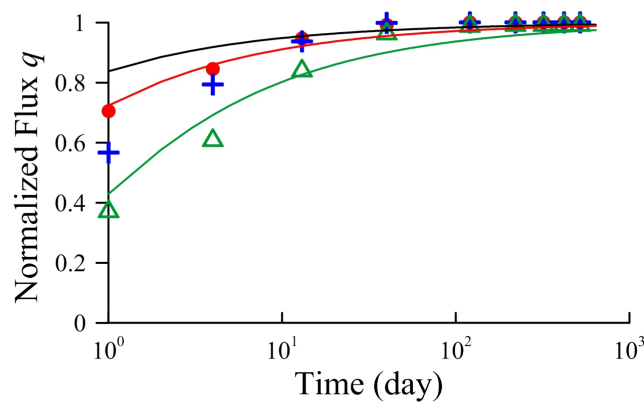

Figure 2. The normalized, ensemble average of transient flux for DFNs with the number off ractures being 20 (a), 60 (b) and 100 (c). The matric conductivity is $K=1 \times 10^{-7} \mathrm{~m} / \mathrm{s}$. Solid lines are the best-fit solutions of the SDE (1). In (a), $D=2 \times 10^{2}, 5 \times 10^{3}$, and $1 \times 10^{4} \mathrm{~m}^{2} / \mathrm{s}$ for the green, red, and black lines, respectively. In (b), $D=2 \times 10^{3}, 1 \times 10^{4}$, and $3 \times 10^{4} \mathrm{~m}^{2} / \mathrm{s}$ for the green, red, and black lines, respectively. In (c), $D=1 \times 10^{6} \mathrm{~m}^{2} / \mathrm{s}$. 
(a) Flux with 20 fractures in a semi-log plot

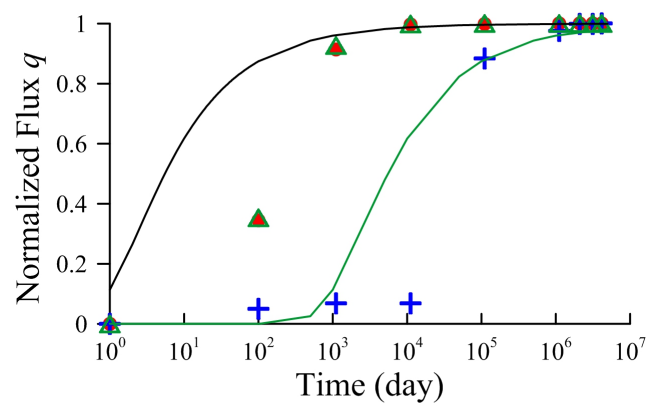

(c) 100 fractures

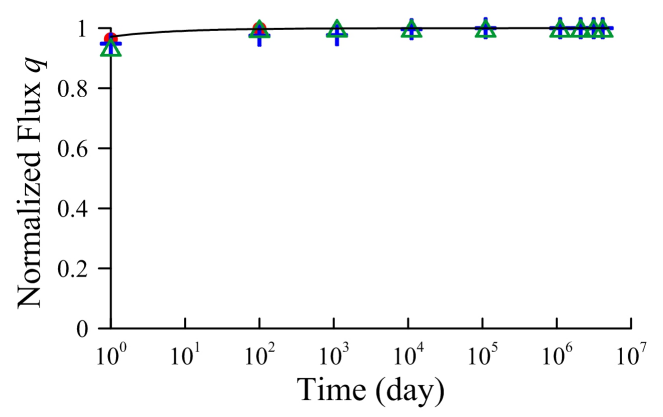

(b) 60 fractures

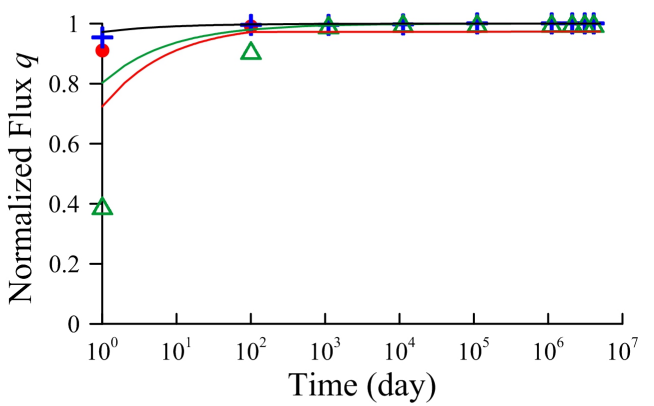

Figure 3. The same as Figure 2, except that the hydraulic conductivity for the rock matrix is $K=1 \times 10^{-8} \mathrm{~m} / \mathrm{s}$. The solid lines are the best-fit solutions of the SDE (1). In (a), $D=5 \times 10^{-1}$ and $5 \times 10^{2} \mathrm{~m}^{2} / \mathrm{s}$ for the green and black lines, respectively. In (b), $D=1 \times$ $10^{6} \mathrm{~m}^{2} / \mathrm{s}$ for the black line. In (c), $D=1 \times 10^{6} \mathrm{~m}^{2} / \mathrm{s}$ for the black line.

Figure 4 shows the distribution of hydraulic head, and Figure 5 lists the probability density function (PDF) for the flux for grids at the outlet boundary.

\section{Discussion}

\subsection{Fracture Density and Hydraulic Gradient Affect Non-Darcian Flow}

The fracture density may affect Non-Darcian flow in field-scale fractured networks by generating complex flow paths for water, which can change with the magnitude of the general hydraulic gradient $J$. For a dense DFN (i.e., the DFN with 100 fractures, see Figure 4(c)), the random distribution of multiple fractures causes a complex flow field consisting of multiple flow zones and surrounding dead ends, especially for a relatively small $J$. The resultant longitudinal ensemble flux may not be as large as that predicted by the Darcy's law (see the dots below the linear trendline shown in Figure 1(c)). The overall hydraulic connectivity along the longitudinal direction can be enhanced with an increasing $J$, leading to a larger longitudinal flux. When the general hydraulic gradient reaches a threshold $J_{s}$, the overall flow paths reach stable (or reach the capacity of connection), and the corresponding longitudinal flux is now mainly controlled by $J$, resulting in Darcian flow.

For a sparse DFN (such as the one with 20 fractures shown in Figure 4(a)), the flow channeling behavior is stronger than that in the dense DFN (similar to 


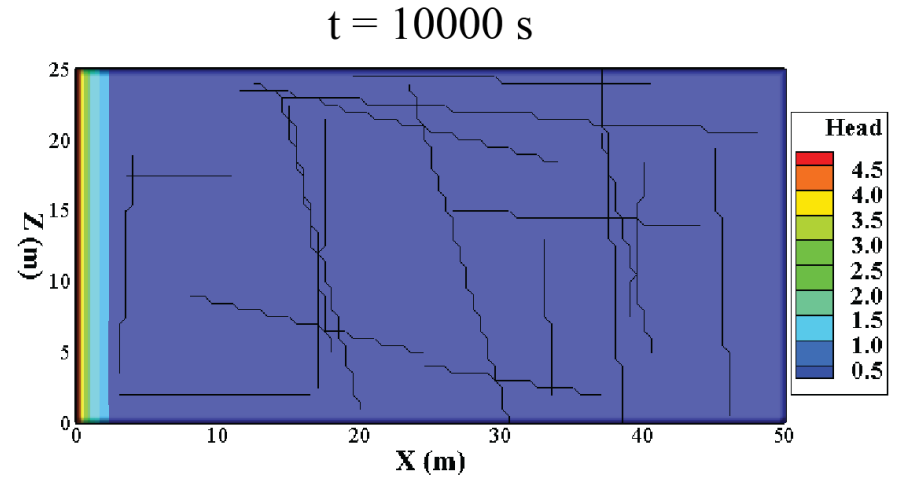

(a)

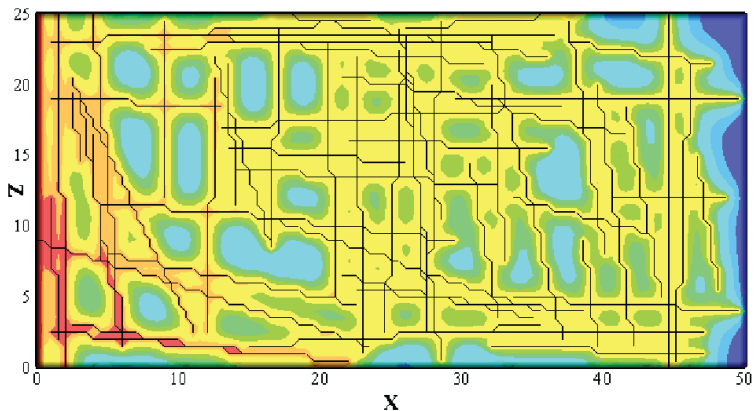

(b)

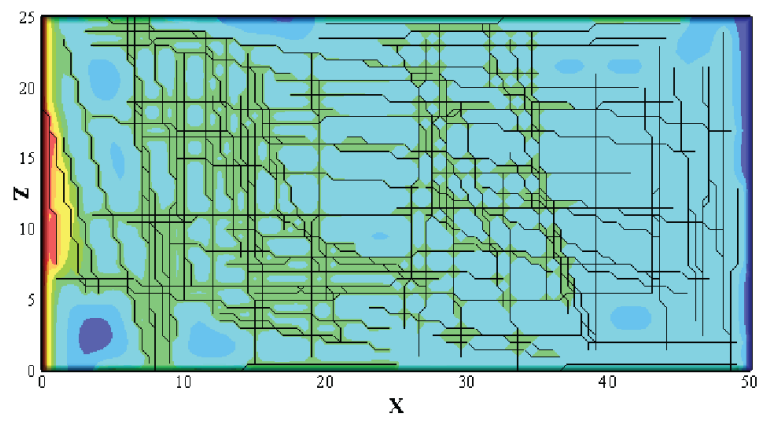

(c) $\mathrm{t}=70000 \mathrm{~s}$

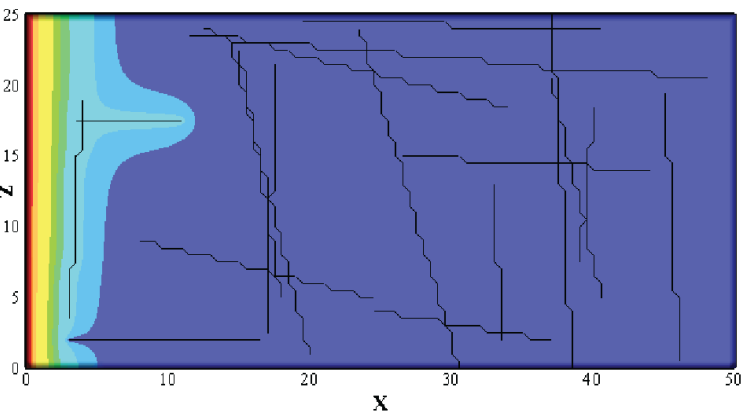

(d)

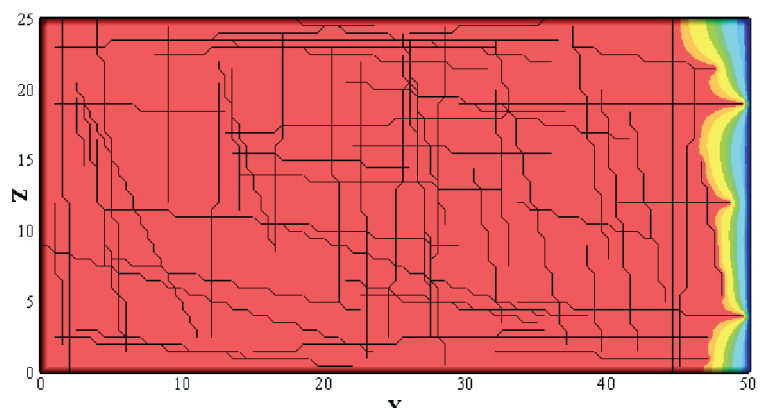

(e)

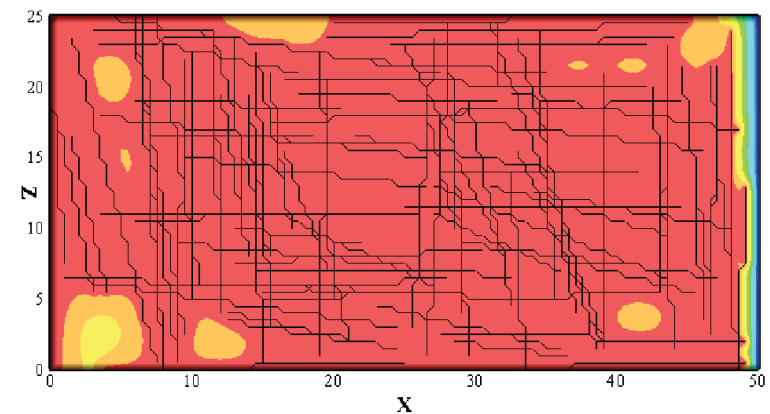

(f)

Figure 4. One realization of the hydraulic head distribution with the matrix hydraulic conductivity $K=1 \times 10^{-7} \mathrm{~m} / \mathrm{sand}$ hydraulic gradient $J=0.1$ at a nearly time $\left(t=1 \times 10^{4} \mathrm{~s}\right)$ for the DFN with $20(\mathrm{a}), 60(\mathrm{~b})$, and 100 fractures (c). The right plot shows a late time ( $\left.t=7 \times 10^{4} \mathrm{~s}\right)$ hydraulic head for the DFN with 20 (d), 60 (e), and 100 fractures (f).

that observed in a fluvial setting with a small proportion of high-permeability ancient channels [18]), and hence it requires a relatively smaller $J_{s}$ to reach the connection capacity and the Darcian flow regime. Therefore, if $J$ is less than $J_{s}$, both the DFN's internal architecture and the pressure gradient affect water flux, generating strong non-Darcian flow. When $J$ is larger than $J_{s}$, Darcian flow dominates all DFNs. The denser for the DFN, the larger for the threshold $J_{s}$. For example, Monte Carlo simulations of this study show that $J_{s}$ is equal to $1 \times 10^{-4}$, $2 \times 10^{-4}$, and $1 \times 10^{-3}$ for the DFN with 20,60 and 100 fractures (see Figure 1 ). The general hydraulic gradient $J$ usually ranges between $10^{-4}$ and $10^{-1}$ in natural aquifers, and hence flow in the field-scale DFN most likely follows Darcy's law, especially for the DFN with sparse fractures. 
The channeling of flow can also be found in Figure 5. Grid-based flux differs significantly between DFNs and shows a broad distribution. The dense DFN has a positive skewness for the PDF (Figure 5(c)), while the sparse DFN has a relatively negative skewness for the PDF (Figure 5(a)), implying a stronger channeling effect for the sparse DFN (where the large flux is embedded in a smaller number of fractures).

\subsection{Impact of Fracture Density on Non-Fickian Pressure Propagation}

The sparse DFN exhibits stronger non-Fickian pressure propagation, especially at the early time, due to the following three reasons. First, the sparse DFN has a relatively small effective hydraulic conductivity, resulting in an overall slow motion for water. It therefore takes a longer time for the transient flux in the spare DFN to reach its asymptote, generating the transient flux with a delayed arriving limb at the early time (see Figure 2 and Figure 3). Second, the inlet boundary of the sparse DFN has a lower probability of direct connection with the fracture than the dense DFN, and hence water must pass through the low-permeable rock matrix before reaching the preferential flow paths. Third, the sparse DFN has a stronger channeling impact, as mentioned above, and hence the major conduits consisting of the sparse and long fractures can transfer water (or pressure) quickly, when water reaches these water "conduits" (see Figure 4). The transient flux can now increase quickly, as shown by the late-time symbols above

(a) Flux with 20 fractures

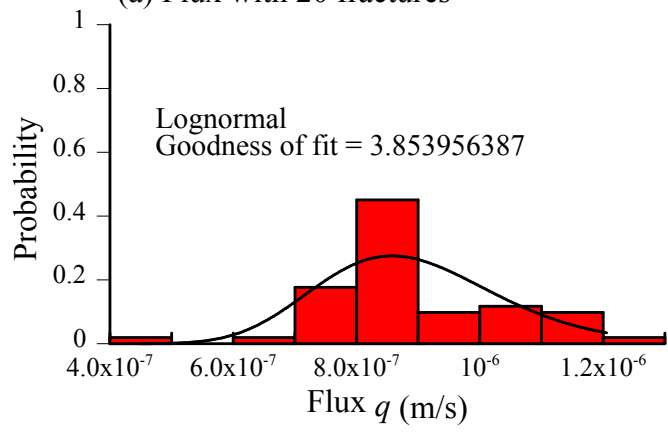

(c) Flux with 100 fractures

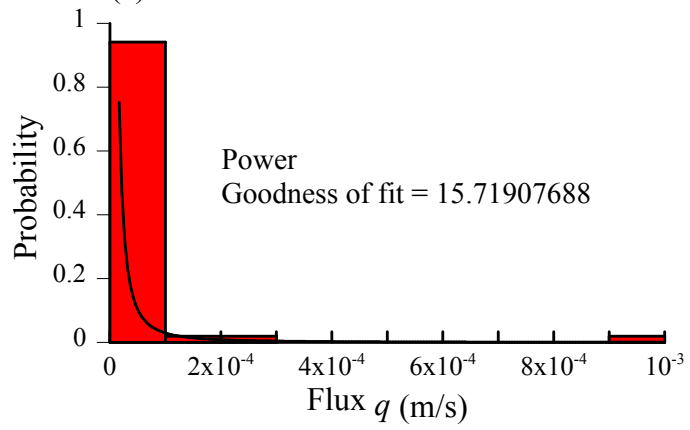

(b) Flux with 60 fractures

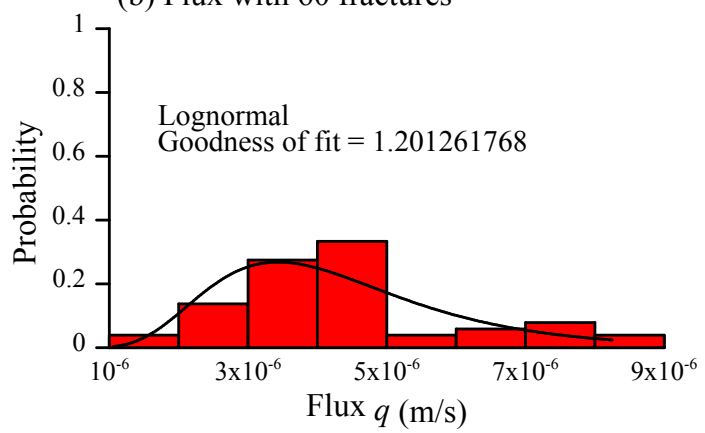

Figure 5. Histogram of the flux of 100 realizations for each node at the outlet boundary for the DFN with 20 (a), 60 (b), and 100 fractures (c). The matrix hydraulic conductivity $K=1 \times 10^{-7} \mathrm{~m} / \mathrm{s}$. The curve represents the best-fit probability distribution function. 
the SDE curve in Figure 2(a). The delayed pressure propagation at the early time and the enhanced propagation at the middle to late times for the sparse DFN, therefore, transfer the hydraulic pressure quite differently from that predicted by a wave diffusive model like the SDE (1).

The delayed transfer of water at the early time is also observed for dense DFNs (Figure 2(c) and Figure 3(c)). Water needs to fill most of the discrete fractures, where some of them are not connected with the major flow paths. The time required to build the major flow paths may decrease with increasing fracture density, since more fractures in the domain can provide a higher probability for an interconnected network. We name this time the "initial regime", where the propagation of pressure does not follow the Fick's law. As shown by the above Monte Carlo simulations, the initial regime is shorter for a denser DFN.

\subsection{Impact of Matrix Permeability on Transient Flow}

Decrease of the rock matrix hydraulic conductivity tends to retard further the propagation of pressure for all DFNs tested in this study, implying that a larger permeability contrast between fractures and matrix leads to a stronger non-Fickian propagation of the hydraulic pressure. It is also noteworthy that for the sparse DFN (with 20 fractures), the inlet boundary for some realizations may not be directly connected with the major fractures, and hence the decrease of the matrix hydraulic conductivity causes significantly slow arrival of the transient flux (by comparing Figure 2(a) and Figure 3(a)). The delayed flow for the other DFNs is not so apparent, since their inlet boundary has a higher chance of fracture connection, as shown by Figure 4(b) \& Figure 4(c).

\subsection{Non-Darcian Flow versus Non-Fickian Pressure Propagation}

We do not find direct correlation between non-Darcian flow and non-Fickian pressure propagation in field-scale DFNs. Non-Darcian flow quantifies the steady-state flux, while non-Fickian pressure propagation focuses on the evolution dynamics of transient flux before reaching its steady-state asymptote. Hence, they need not to be directly connected. Indeed, the above Monte Carlo simulations showed that the dense DFN with strong non-Darcian flow tends to exhibit weak non-Fickian pressure propagation. Our analysis also shows that the threshold hydraulic gradient $J_{s}$ distinguishes Darcian and non-Darcian flow, while the initial regime related to fracture connectivity and architecture affects the early-time non-Fickian pressure propagation.

\section{Conclusions}

This study conducts Monte Carlo simulations to identify possible non-Darcian flow and non-Fickian pressure propagation in field-scale discrete fracture networks. Multiple scenarios of DFNs are generated with different fracture densities and matrix hydraulic conductivities. Flux needed for Darcian/non-Darcian flow analysis is calculated from the steady-state flow models using HGS, and the 
transient motion of water is modeled to reveal possible non-Fickian propagation of hydraulic pressure. Numerical simulations and result analysis lead to the following three main conclusions.

First, both the fracture network architecture and the general hydraulic gradient $J$ affect the Darcian/non-Darcian flow in DFNs. The fracture density may affect flow dynamics by generating complex flow paths for water, and the gradient Jcan adjust the flow field and provide the criterion for Darcian/non-Darcian flow. Strong non-Darcian flow appears for $J$ less than the threshold $J_{s}$, where this threshold increases with an increasing fracture density.

Second, fracture density affects significantly the propagation of hydraulic head or pressure in the DFN. A sparse DFN can cause both delayed motion of water at the early time (likely due to the small effective hydraulic conductivity and the poor fracture connectivity) and enhanced flow at the middle/late time (likely due to the enhanced flow channeling), resulting in strong non-Fickian pressure propagation.

Third, the non-Darcian flow pattern needs not to be directly related to the non-Fickian pressure propagation process in field-scale DFNs, because they refer to different states of water flow and their controlling factors may not be exactly the same.

\section{Acknowledgements}

This work was funded partially by the National Natural Science Foundation of China under grants 41628202, 41330632 and 11572112. This paper does not necessarily reflect the views of the funding agency.

\section{References}

[1] Darcy, H. (1856) Les fontainespubliques de la ville de Dijon: exposition et application. Victor Dalmont.

[2] Kohl, T., Evans, K.F., Hopkirk, R.J., Jung, R. and Rybach, L. (1997) Observation and Simulation of Non-Darcian Flow Transients in Fractured Rock. Water Resources Research, 33, 407-418. https://doi.org/10.1029/96WR03495

[3] Şahin, A.U. (2018) A Particle Swarm Optimization Assessment for the Determination of Non-Darcian Flow Parameters in a Confined Aquifer. Water Resources Management, 32, 751-767. https://doi.org/10.1007/s11269-017-1837-9

[4] Holditch, S.A. and Morse, R.A. (1976) The Effects of Non-Darcy Flow on the Behavior of Hydraulically Fractured Gas Wells. Journal of Petroleum Technology, 28, 1169-1179. https://doi:10.2118/5586-PA

[5] Miskimins, J.L., Lopez, H.D.J. and Barree, R.D. (2005) Non-Darcy Flow in Hydraulic Fractures: Does It Really Matter? SPE Annual Technical Conference and Exhibition. https://doi.org/10.2118/96389-MS

[6] Wen, Z., Huang, G. and Zhan, H. (2006) Non-Darcian Flow in a Single Confined Vertical Fracture toward a Well. Journal of Hydrology, 330, 698-708. https://doi.org/10.1016/j.jhydrol.2006.05.001

[7] Hunt, M.L. and Tien, C.L. (1990) Non-Darcian Flow, Heat and Mass Transfer in Catalytic Packed-Bed Reactors. Chemical Engineering Science, 45, 55-63. 
https://doi.org/10.1016/0009-2509(90)87080-C

[8] Liu, R., Li, B. and Jiang, Y. (2016) Critical Hydraulic Gradient for Nonlinear Flow through Rock Fracture Networks: The Roles of Aperture, Surface Roughness, and Number of Intersections. Advances in Water Resources, 88, 53-65. https://doi.org/10.1016/j.advwatres.2015.12.002

[9] Cooke Jr., C.E. (1973) Conductivity of Fracture Proppants in Multiple Layers. Journal of Petroleum Technology, 25, 1-101. https://doi.org/10.2118/4117-PA

[10] Yeo, I.W. and Ge, S. (2001) Solute Dispersion in Rock Fractures by Non-Darcian Flow. Geophysical Research Letters, 28, 3983-3986. https://doi.org/10.1029/2001GL013274

[11] Therrien, R., McLaren, R.G., Sudicky, E.A. and Panday, S.M. (2010) HydroGeoSphere: A Three-Dimensional Numerical Model Describing Fully-Integrated Subsurface and Surface Flow and Solute Transport. Groundwater Simulations Group, University of Waterloo, Waterloo, ON.

[12] Long, J.C.S., Remer, J.S., Wilson, C.R. and Witherspoon, P.A. (1982) Porous Media Equivalents for Networks of Discontinuous Fractures. Water Resources Research, 18, 645-658. https://doi.org/10.1029/WR018i003p00645

[13] Lu, B., Zhang, Y., Reeves, D.M., Sun, H. and Zheng, C. (2018) Application of Tempered-Stable Time Fractional-Derivative Model to Upscale Subdiffusion for Pollutant Transport in Field-Scale Discrete Fracture Networks. Mathematics, 6, 5. https://doi.org/10.3390/math6010005

[14] McKenna, S.A., Meigs, L.C. and Haggerty, R. (2001) Tracer Tests in a Fractured Dolomite: 3. Double-Porosity, Multiple-Rate Mass Transfer Processes in Convergent Flow Tracer Tests. Water Resources Research, 37, 1143-1154. https://doi.org/10.1029/2000WR900333

[15] Fiori, A. and Becker, M.W. (2015) Power Law Breakthrough Curve Tailing in a Fracture: The Role of Advection. Journal of Hydrology, 525, 706-710. https://doi.org/10.1016/j.jhydrol.2015.04.029

[16] Hornberger, G.M., Ebert, J. and Remson, I. (1970) Numerical Solution of the Boussinesq Equation for Aquifer-Stream Interaction. Water Resources Research, 6, 601-608. https://doi.org/10.1029/WR006i002p00601

[17] Munson, B.R., Okiishi, T.H., Rothmayer, A.P. and Huebsch, W.W. (2014) Fundamentals of Fluid Mechanics. John Wiley \& Sons, Hoboken.

[18] Zhang, Y., Green, C.T. and Baeumer, B. (2014) Linking Aquifer Spatial Properties and Non-Fickian Transport in Mobile-Immobile Like Alluvial Settings. Journal of Hydrology, 512, 315-331. https://doi.org/10.1016/j.jhydrol.2014.02.064 